NOTES

\title{
Shape of Three-Phase Coexistence Curve near the Critical End Points for Four-Component Homologous Polystyrene Solution
}

\author{
Masato SuZukI, Rio KITA, ${ }^{\dagger}$ Toshiaki DoBASHI, ${ }^{\dagger \dagger}$ and Mitsuo NAKATA* \\ Department of Biological and Chemical Engineering, Faculty of Engineering, \\ Gunma University, Kiryu, Gunma 376-8515, Japan \\ *Department of Polymer Science, Faculty of Science, Sapporo 060-0810, Japan
}

(Received November 26, 1999)

\begin{abstract}
KEY WORDS Coexistence Curve / Three-Phase Equilibrium / Critical Exponent / Upper Critical End Point/Lower Critical End Point/
\end{abstract}

Solutions of homologous polymers with different molecular weights in a solvent exhibits various aspects of phase equilibrium with increase of the polymer components. For multi-component systems the total polymer volume fraction $\phi_{s}$ in each phase can be measured accurately by a refractive index method and the coexistence curve can be obtained on a diagram of temperature versus $\phi_{\mathrm{s}}$ in high precision, because the refractive index of the homologous polymers is independent of molecular weight. Free energy for the multi-component system can be determined experimentally on the basis of the FloryHuggins equation. We can predict phase equilibrium behavior by analysis of the free energy and carry out experiments accordingly. ${ }^{1}$

For binary systems of monodisperse polystyrene(PS) in methylcyclohexane $(\mathrm{MCH})$ we determined the critical exponent $\beta$ and showed the molecular weight dependence of the range of the simple scaling. ${ }^{2,3}$ For ternary systems of two PS homologs(PSI + PSII) in MCH we measured the three-phase coexistence curve and analyzed the behavior of the diameters and the shape of the coexistence curve near the upper critical end point (UCEP) and lower critical end point(LCEP)., ${ }^{4,5}$ Recently, we observed a reentrant three-phase equilibrium for the quaternary system of PSI + PSII + PSIII $+\mathrm{MCH}$ as predicted from a numerical analysis of the empirical free energy. ${ }^{6}$ It is interesting to explore unchanged behavior and new aspects of the phase equilibrium, when the homologous polymer solution changes from the ternary to quaternary system. In this study we measured the three-phase coexistence curve for the quaternary system to compare it with that for the ternary system and to disclose the effect of the component PSIII on the threephase coexistence curve. For the ternary system, the entire three-phase coexistence curve can be observed for a solution, whose composition is specified by a point on the Gibbs composition triangle in a space of volume fraction of PS I $\left(\phi_{1}\right)$-volume fraction of PS II $\left(\phi_{2}\right)$, since the degree of freedom of the three-phase equilibrium of the system is one at atmosphere. This made the coexistence curve measurement near the top (the critical endpoints) very difficult. ${ }^{4}$ For quaternary system the compositions of the solution, which yields the entire three-phase coexistence curve, is given by a line in the composition tetrahedron in $\phi_{1}-\phi_{2}-\phi_{3}$ space. This relaxes the restriction on the entire three-phase coexistence curve measurements. Here, $\phi_{1}, \phi_{2}$, and $\phi_{3}$ are the volume fractions of PS I, PS II, and PS III, respectively. In fact we could locate the three-phase region easily for the present quaternary system.

\section{EXPERIMENTAL}

Polystyrene with the molecular weight $1.81 \times 10^{4}$ $\left(M_{w} / M_{n}=1.01\right)(\mathrm{PSI})$ and $70.6 \times 10^{4}\left(M_{w} / M_{n}=1.05\right)$ (PSII) were purchased from TOSOH Co., Tokyo and used without further fractionation, where $M_{w}$ and $M_{n}$ are the weight- and number-average molecular weight, respectively. PSIII was prepared by solution fractionation from PS sample provided by Japan Synthetic Rubber Co., Ltd. The molecular weight of PSIII was determined as $M_{w}=$ $2.0 \times 10^{7}$ by a novel plot of observed light scattering data. ${ }^{7}$ Methylcyclohexane was distilled twice after being passed through silica gel. The coexistence curve was measured by the refractive index method in a water bath, the temperature of which was controlled within $\pm 0.002 \mathrm{~K}$. The solution for the measurement had the overall volume fractions of PSI, PSII, and PSIII given by $0.1698,0.00930$ and $1.55 \times 10^{-4}$, respectively. Detailed procedure of the experiment was reported in the previous paper. ${ }^{2,8}$

\section{RESULTS AND DISCUSSION}

Figure 1 exhibits the observed coexistence curve for the present four-component system in a diagram of temperature $T\left({ }^{\circ} \mathrm{C}\right)$ versus total volume fraction $\phi_{\mathrm{s}}$ of PS. With decreasing temperature the solution begins to separate into two phases at the cloud-point temperature $32.27^{\circ} \mathrm{C}$. The points $\mathrm{C}$ and $\mathrm{C}^{\prime}$ are the cloud point and conjugate point, respectively. Near $24.3^{\circ} \mathrm{C}$ the dilute phase starting from the cloud point $\mathrm{C}$ separates into the dilute phase $\alpha$ and intermediate phase $\beta$, which gives rise to three-phase equilibrium together with the concentrated

\footnotetext{
${ }^{\dagger}$ Present address: The Institute of Physical and Chemical Research (RIKEN), Wako, Saitama 351-0198, Japan

${ }^{\dagger}{ }^{\dagger}$ To whom all correspondence should be addressed.
} 


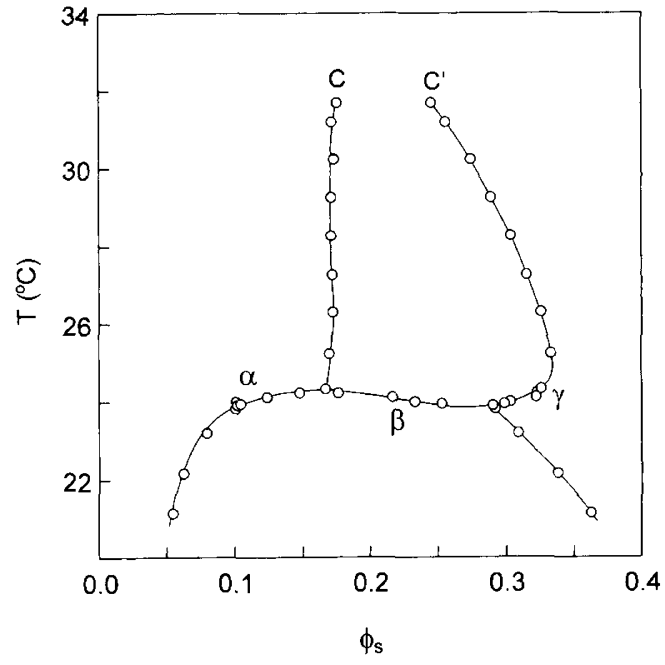

Figure 1. Coexistence curve observed for the quaternary system PSI + PSII + PSIII + methylcyclohexane in diagram of total polystyrene(PS) volume fraction $\phi_{\mathrm{s}}$ versus temperature $T$. Points $\mathrm{C}$ and ' $\mathrm{C}^{\prime}$ are the cloud point and the conjugate point, respectively. $\alpha$, $\beta$, and $\gamma$ denote the three branches in the three-phase equilibrium.

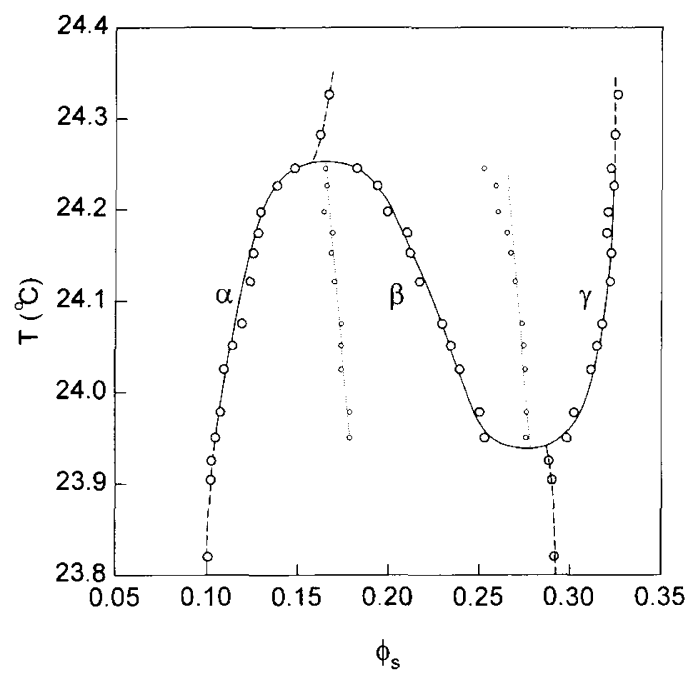

Figure 2. Enlarged three-phase region in Figure 1. Solid and broken lines represent the three-phase and two-phase coexistence curves, respectively. The dotted lines give diameters for adjacent phases in three-phase equilibrium.

phase $\gamma$ starting the conjugate point $\mathrm{C}^{\prime}$. Near $23.9^{\circ} \mathrm{C}$ the intermediate and concentrated phases coalesce into one phase and two phase equilibrium occurs again. Figure 2 shows the coexisting curve near the three-phase region in the diagram of Figure 1 with enlarged temperature scale. The solid and broken lines represent the threephase and two-phase coexistence curves, respectively. The three-phase coexistence curve is s-shaped with a maximum and a minimum, which may give UCEP and LCEP, respectively. The small open circles give the diameters for the adjacent two phases. The diameter for the $\alpha$ and $\beta$ phases is well represented by a straight line in the whole range, while the diameter for the $\beta$ and $\gamma$ phases shows deviation from the straight line at higher temperatures. It should be noted that the straight lines are given parallel each other. The intersection of the

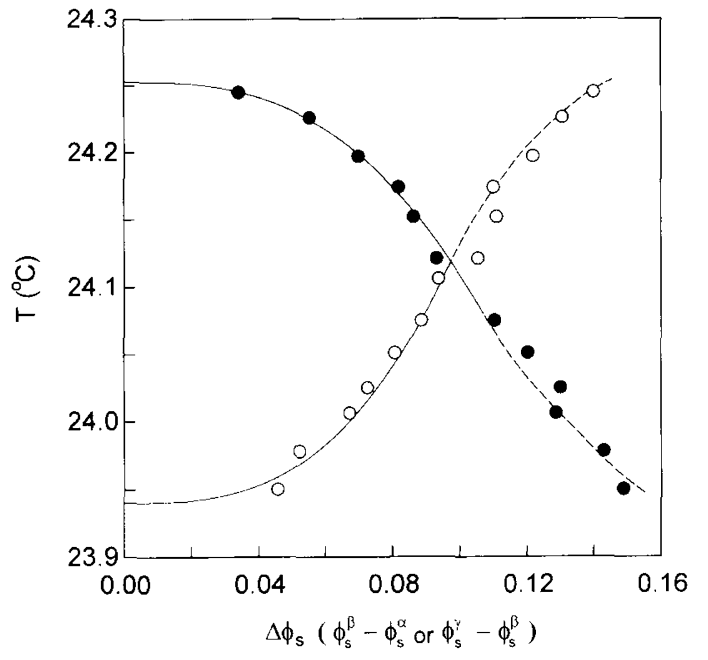

Figure 3. Plots of temperature T versus volume fraction difference $\Delta \phi_{\mathrm{s}}$ for total polystyrene between two adjacent phases in three-phase equilibrium. The filled and open circles represent data for upper and lower critical end points, respectively. The solid lines are given by eq 1 with the obtained values of $\beta$ and $B$.

three-phase coexistence curve and diameter can be used to determine the critical end point. This behavior of the diameters is very similar to that for the threecomponent system of PSI + PSII + MCH. ${ }^{5}$ Figure 3 shows plots of the composition difference between the two adjacent phases $\Delta \phi_{\mathrm{s}}$ versus temperature, where the filled and open circles give the differences between the $\alpha$ and $\beta$ phases and between $\beta$ and $\gamma$ phases, respectively. The difference near the critical end point may be represented by the relation,

$$
\Delta \phi_{\mathrm{s}}=B(\Delta T)^{\beta}
$$

where $\Delta T$ denotes the temperature distance from the UCEP temperature $T_{\mathrm{U}}$ or LCEP temperature $T_{\mathrm{L}}$. Assuming various values of $T_{\mathrm{U}}$ and $T_{\mathrm{L}}$ eq 1 was fitted to the data points by the nonlinear least-squares method. For the data points near UCEP the standard deviation of the fit became a minimum for $T_{\mathrm{U}}=24.253^{\circ} \mathrm{C}$, at which $\beta_{\mathrm{U}}$ $=0.366 \pm 0.016$ and $B_{\mathrm{U}}=0.203 \pm 0.008$ were determined. Similarly, we obtained $T_{\mathrm{L}}=23.940^{\circ} \mathrm{C}, \beta_{\mathrm{L}}=$ $0.340 \pm 0.042$ and $B_{\mathrm{L}}=0.175 \pm 0.017$ for the data points near LCEP. The solid lines in Figure 3 were calculated by eq 1 with the above values of $\beta$ and $B$.

In the previous study, ${ }^{4,5}$ we analyzed the three-phase coexistence curve for the ternary system $\operatorname{PSI}(M=1.73$ $\left.\times 10^{4}\right)+\operatorname{PSII}\left(M=7.19 \times 10^{5}\right)+\mathrm{MCH}$ in the same manner and obtained $\beta_{\mathrm{U}}=0.381 \pm 0.012$ and $B_{\mathrm{U}}=$ $0.202 \pm 0.005$ at UCEP and $\beta_{\mathrm{L}}=0.342 \pm 0.031$ and $B_{\mathrm{L}}$ $=0.153 \pm 0.013$ at LCEP. According to the calculation using a generalized Flory-Huggins free energy, the tie lines near UCEP were parallel to each other, whereas those near LCEP were not parallel but the surface including a set of tie lines near the LCEP was twisted. From this aspect the value $\beta_{\mathrm{U}}=0.381 \pm 0.012$ was compared with the renormalized exponent $\beta^{\prime}=\beta /(1-\alpha)$ for the ternary system and/or the impurity system, where $\alpha$ denotes the critical exponent for specific heat. ${ }^{9}$ Thus, the critical behavior at UCEP was concluded to be the same 
as that near the usual plait point of the three-component system or the impurity system. The smaller value $\beta_{\mathrm{L}}=$ $0.342 \pm 0.031$ was ascribed to the improper behavior of the tie line. The present values of $\beta$ and $B$ for the quaternary system agree with previous values for the ternary system within the experimental errors both for UCEP and LCEP. To reveal the correspondence between the ternary and quaternary systems, we calculated the tie lines near the critical end points using a generalized Flory-Huggins free energy with an empirically determined interaction parameter. ${ }^{3}$ Near UCEP the tie lines calculated at different temperature were roughly parallel to each other in a composition tetrahedron, while near LCEP the tie line changed direction noticeably with temperature. Thus, the three-phase behavior of the quaternary system agrees with that of the ternary system. This agreement confirms typical behavior of the threephase equilibrium in the multi-component homologous polymer system, though the three-phase coexistence curve cannot be determined reliably because of its appearance in a very narrow range. Since the overall volume fraction of PSIII is small compared with those of PSI and PSII, the three-phase equilibrium is formed near the surface of the composition triangle for PSI, PSII, and $\mathrm{MCH}$ of a composition tetrahedron. However, this small fraction is large enough to bring about an essential change in phase behavior of the quaternary system on account of the large molecular weight. We observed a reentrant three-phase equilibrium for the present quaternary system with overall volume fractions $0.1875,0.00360$ and $1.430 \times 10^{-4}$ for PSI, PSII, and
PSIII, respectively: On lowering temperature, phase behavior changed as one-phase, two-phase, three-phase, two-phase, three-phase, and two-phase equilibrium as predicted from a numerical analysis of the free energy. ${ }^{6}$ It should be noted that the volume fraction of PSIII is comparable with that of the present quaternary system. It is interesting to explore unchanged behavior and newly emerging aspects in phase equilibrium with increase in polymer components in homologous polymer systems.

Acknowledgments. We are grateful to Mr. Y. Mikawa for his technical assistance. This work was partly supported by a Grant-in-Aid from Ministry of Education, Science, Sports and Culture under Grant number 11640385

\section{REFERENCES}

1. H. Fujita, "Polymer Solutions", Elsevier, Amsterdam, 1990.

2. T. Dobashi, M. Nakata, and M. Kaneko, J. Chem. Phys., 72, 6685 (1980).

3. T. Dobashi, M. Nakata, and M. Kaneko, J. Chem. Phys., 72, 6692 (1980)

4. T. Dobashi and M. Nakata, J. Chem. Phys., 84, 5775 (1986).

5. M. Nakata and T. Dobashi, J. Chem. Phys., 84, 5782 (1986).

6. M. Suzuki, T. Dobashi, Y. Mikawa, K. Yamamura, and M. Nakata, J. Phys. Soc. Jpn., in press.

7. M. Nakata, Polymer, 38, 9 (1997).

8. R. Kita, T. Dobashi, T. Yamamoto, M. Nakata, and K. Kamide, Phys. Rev. E, 55, 3159 (1997).

9. M. E. Fisher, Phys. Rev., 176, 257 (1968). 